\title{
Study on the array calibration algorithm based on the minimum mean square error criterion ${ }^{1}$
}

\author{
Huanhui Liang ${ }^{1, a^{*}}$, Chao Tang ${ }^{2, b}$, Biyuan Jiang ${ }^{1, c}$, Bin fang ${ }^{1, d}$ \\ ${ }^{1}$ Guangzhou Vocational College of Science and Technology, GuangZhou, China \\ ${ }^{2}$ Guangzhou College of Technology and Business

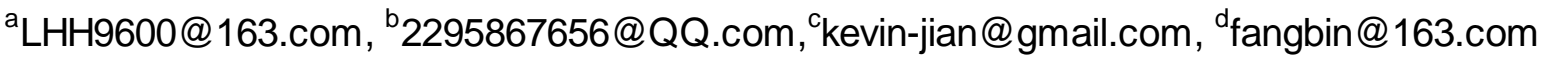

Keywords: Antenna Array, Calibration matrix, Wireless Communication System, MMSE

Abstract: The antenna array could raise the performance of the wireless communication system. There always exists certain error between the system theoretical output and actual output due to the system error. Based on the minimum mean square error criterion, we introduce an antenna array calibration matrix which could reduce the system error effectively.

\section{Introduction}

In the modern wireless mobile communication system, as the increasing of user amount, the system performance decreased. The study on the antenna arrays shows that the introduction of antenna arrays could improve the performance of the system, such as signal to noise ratio, system capacity, etc.[1.2]. For the antenna array, the output signal is composed of signals from different array elements, and the corresponding coefficients are dynamically changed.

For the uplink and downlink of the antenna array, with the surrounding environment changes, there exists error in array element coefficients, which may be caused by mutual coupling, temperature variation, electronic component aging and so on. For the uplink and downlink transmit, the error in element coefficients is not the same, The calibration for the uplink and downlink transmit antenna array must be done separately.[3]. Assumed that the error has been calibrated for the uplink weight vector, the array vector for the downlink beamforming may be calibrated according to the array coefficients for uplink. It just need to calibrate the error in the downlink vector. The calibration of the array coefficients could improve the quality of the communication system, increase the system capacity and other aspects of the performance [4,5].

In this paper, the calibration of the array vector based on MMSE criterion is studied.

\section{Problem description}

Assumed that the antenna array has $\mathrm{N}$ elements, the interval between the array elements is one half of the signal wavelength. For the signal with incident angle $\theta_{m}$, the ideal received signal is:

$$
\mathbf{x}_{m}=\alpha_{m} \boldsymbol{\alpha}_{m}
$$

Where $\alpha_{m}$ is the channel gain, ${ }^{\mathbf{a}_{m}}$ is the steering vector for the direction of $\theta_{m}$.

In fact, the actual received signal should be:

$$
\mathbf{y}_{m}=\mathbf{B} \mathbf{x}_{m}+\mathbf{e}_{m}
$$

$\mathbf{B}$ is the error matrix of $\mathrm{N} * \mathrm{~N}, \mathbf{e}_{m}$ is the sum of the interference and noise. And $\left\{\mathbf{y}_{1}, \mathbf{y}_{2}, \cdots, \mathbf{y}_{M}\right\}$ is the received signal corresponding to different incident direction $\left\{\theta_{1}, \theta_{2}, \cdots, \theta_{M}\right\}$ respectively. The calibration matrix $\mathbf{C}$ is a matrix of $\mathrm{N} \times \mathrm{N}$. Then

\footnotetext{
${ }^{1}$ This work supported partially by the Science Foundation of GuangZhou Vocational College of Science and Technology(2014ZR08)
} 


$$
S=\sum_{m=1}^{M}\left\|\mathbf{C} \mathbf{y}_{m}-\hat{\mathbf{x}}_{m}\right\|^{2}
$$

Where $\hat{\mathbf{x}}_{m}$ is the estimated value of the ideal received signal to the estimated channel gain $\hat{\alpha}_{m}$.

In order to obtain the optimal calibration matrix, the $\mathrm{S}$ should take the minimum value. And in other words, the matrix $C$ is solved based on minimum mean square error criterion. The optimal calibration matrix $C$ could make the $\mathrm{S}$ get a minimum value, where $\mathrm{S}$ is the mean square error of the estimated signal to the calibrated receiving signal

\section{Description of the calibration algorithm}

In order to calibrate the array coefficients, based on the minimum criterion, a calibration matrix which minimized the mean square error between the array output signal and the ideal signal.

Assumed $\mathbf{C}_{i-1}$ is one estimated value of the channel $\mathbf{C}$, and $\mathbf{z}_{m}=\mathbf{C}_{i-1} \mathbf{y}_{m}$. The estimation of the channel gain $\alpha_{m}$ is :

$$
\hat{\alpha}_{m}=\mathbf{a}_{m}^{H} \mathbf{z}_{m} /\left|\mathbf{a}_{m}\right|^{2}
$$

Given two $\mathrm{N} * \mathrm{M}$ matrix $\hat{\mathbf{X}}$ and $\mathbf{Y}$,

$$
\begin{aligned}
& \hat{\mathbf{X}}=\left(\hat{\mathbf{x}}_{1}, \hat{\mathbf{x}}_{2}, \cdots, \hat{\mathbf{x}}_{M}\right) \\
& \mathbf{Y}=\left(\mathbf{y}_{1}, \mathbf{y}_{2}, \cdots, \mathbf{y}_{M}\right)
\end{aligned}
$$

A new estimate value is obtained by solving the equation.

$$
\mathbf{C}_{i-1} \mathbf{Y}=\mathbf{X}
$$

i.e. $\mathbf{C}_{i-1}=\left(\hat{\mathbf{X}} \hat{\mathbf{X}}^{H}\right)\left(\mathbf{Y} \hat{\mathbf{X}}^{H}\right)^{-1}$

In this paper, we use a neighbor base station antenna with corrected error free to calibrate the base station antenna. For the calibration to the uplink channel, the adjacent base station transmits the reference signal. The reference signal is received by the array elements in turn. Then the received signal is processed and the calibration matrix of uplink channel obtained. For the downlink channel calibration, each array element is transmitted to the reference signal, and the adjacent base station is received by the adjacent base station.

The proposed algorithm is applied to the calibration of the transmitter and receiver antenna array, and a calibration matrix is obtained, which could directly compensate the error of the antenna array both on the uplink and downlink channel.

Without loss of generality, assuming that the antenna array has $\mathrm{N}$ array elements, the interval between array elements is $\lambda / / 2$, the transmitted reference signal is $p$, which with the incident angle $\theta$. For the antenna array, the ideal steering vector is

$$
\mathbf{V}=\left[v_{1}, v_{2}, \cdots, v_{m}, \cdots, v_{N}\right]
$$

where $v_{m}=e^{j *(m-1) * p i * \sin (\theta)}$.

But actually there exits error in steering vector of the system always, in the paper we assume

$$
\hat{\mathbf{V}}=\left[\hat{v}_{1}, \hat{v}_{2}, \cdots, \hat{v}_{m}, \cdots, \hat{v}_{N}\right]
$$

where $\hat{v}_{m}=\beta_{m} e^{j^{*}\left((m-1)^{*} p i^{*} \sin (\theta)+\Delta_{m}\right)}, \beta_{m}$ is the gain error, $\Delta_{m}$ is the direction error. Then the received the signal in array element $\mathrm{M}$ is

$$
r_{m}(t)=\alpha \hat{v}_{m} p_{m}(t)+n_{m}(t)
$$


The channel estimation of the received signal by the array element $\mathrm{M}$ is

$$
\hat{\alpha}_{m}=\frac{1}{S} \sum_{t=1}^{S} v_{m}^{*} r_{m}(t) p_{m}(t)
$$

Where $\mathrm{S}$ is the number of transmitted code, $v_{m}^{*}$ is conjugate number of $v_{m}$.

In the ideal case, the channel estimation of each element should be the same, but in fact he channel estimation for different array element is not the same. For example, assumed the signal in the first array element as the reference signal, the channel estimation of the array element $\mathrm{M}$ is compare to the first array element, and the error of the array element $\mathrm{M}$ is solved:

$$
c_{m}=\hat{\alpha}_{1} / \hat{\alpha}_{m} ; \quad m=1,2, \cdots, N
$$

In order to calibrate the error of the array, the calibration matrix is obtained:

$$
\mathbf{C}=\left(\begin{array}{lll}
c_{1} & & 0 \\
& \ddots & \\
0 & & c_{N}
\end{array}\right)
$$

\section{Simulation results and analysis}

In order to analysis the proposed algorithm, the simulation of the algorithm was made in the Matlab platform. In the simulation, the calibration matrix is obtained according to Eq.8.

Without loss of generality, assumed $\mathrm{M}=4, \Delta^{\prime}=0.0001$, the initial matrix $\mathbf{C}_{0}$ is the $\mathrm{N} \times \mathrm{N}$ unit matrix.

Firstly to analysis the impact of the calibration matrix on the array output, assumed the symbol length is 160 , SNR is $20 \mathrm{~dB}, \mathrm{~N}$ elements and the gain error is random uniform distribution between $0.9 \sim 1$, the range of direction error is $1^{\circ} \sim 120^{\circ}$. range respectively in the error rate. The relative error between ideal array beamfoming and the calibrated array beamforming is $\frac{\|\mathbf{V}-\mathbf{C} \hat{\mathbf{V}}\|}{\|\mathbf{V}\|}$, which is shown in Fig.1.

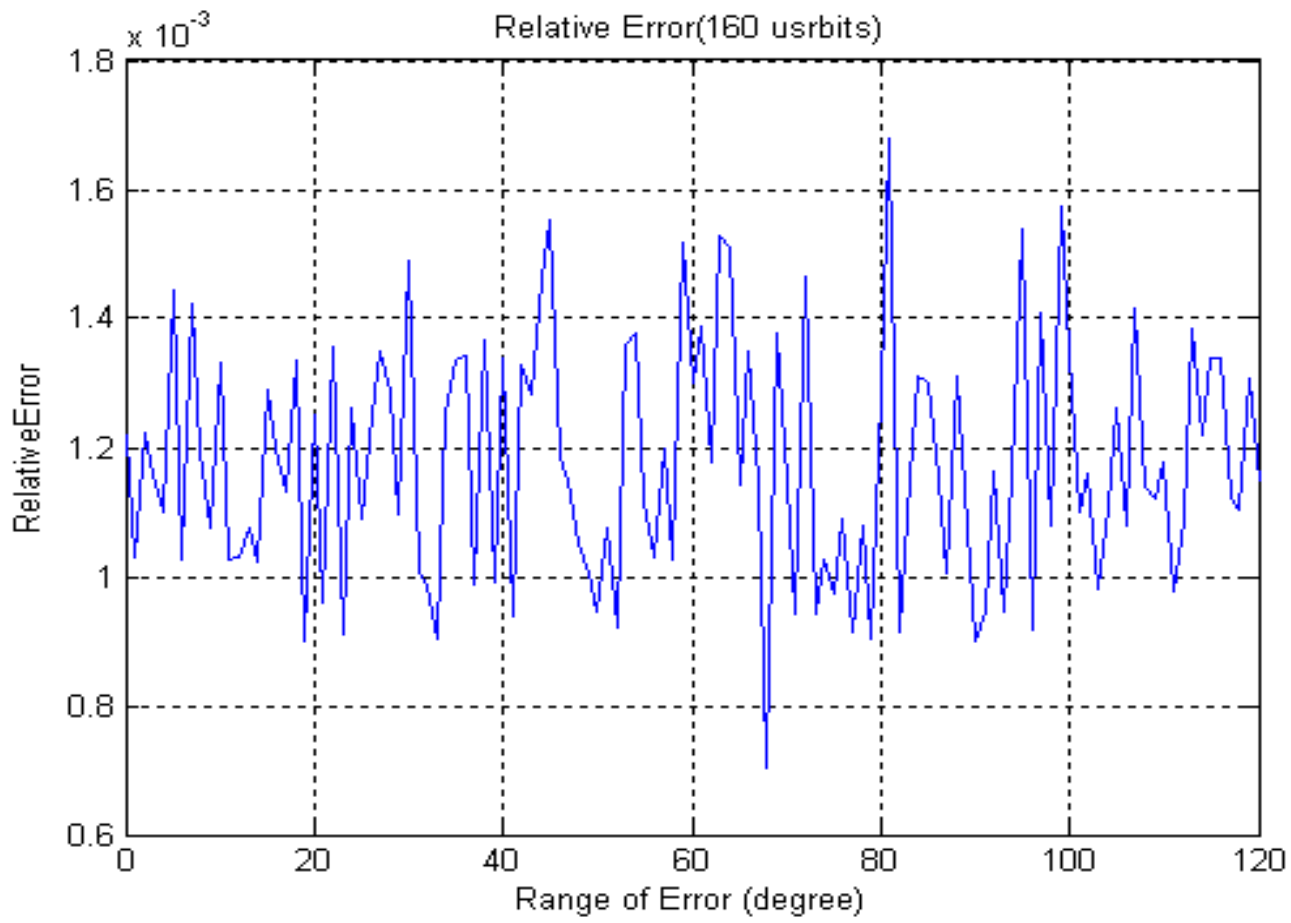

Fig.1. the influence of direction error on relative error 
Secondly, with the calibration matrix, we analysis the influence on the array output while the changing of received signal's SNR. Only the range of direction error is changed to $-50^{\circ} \sim 50^{\circ}$, the other assumption just as in the previous case. In the simulation, the influence of calibration was studied. The relative error according to the change of SNR was shown in Fig.2

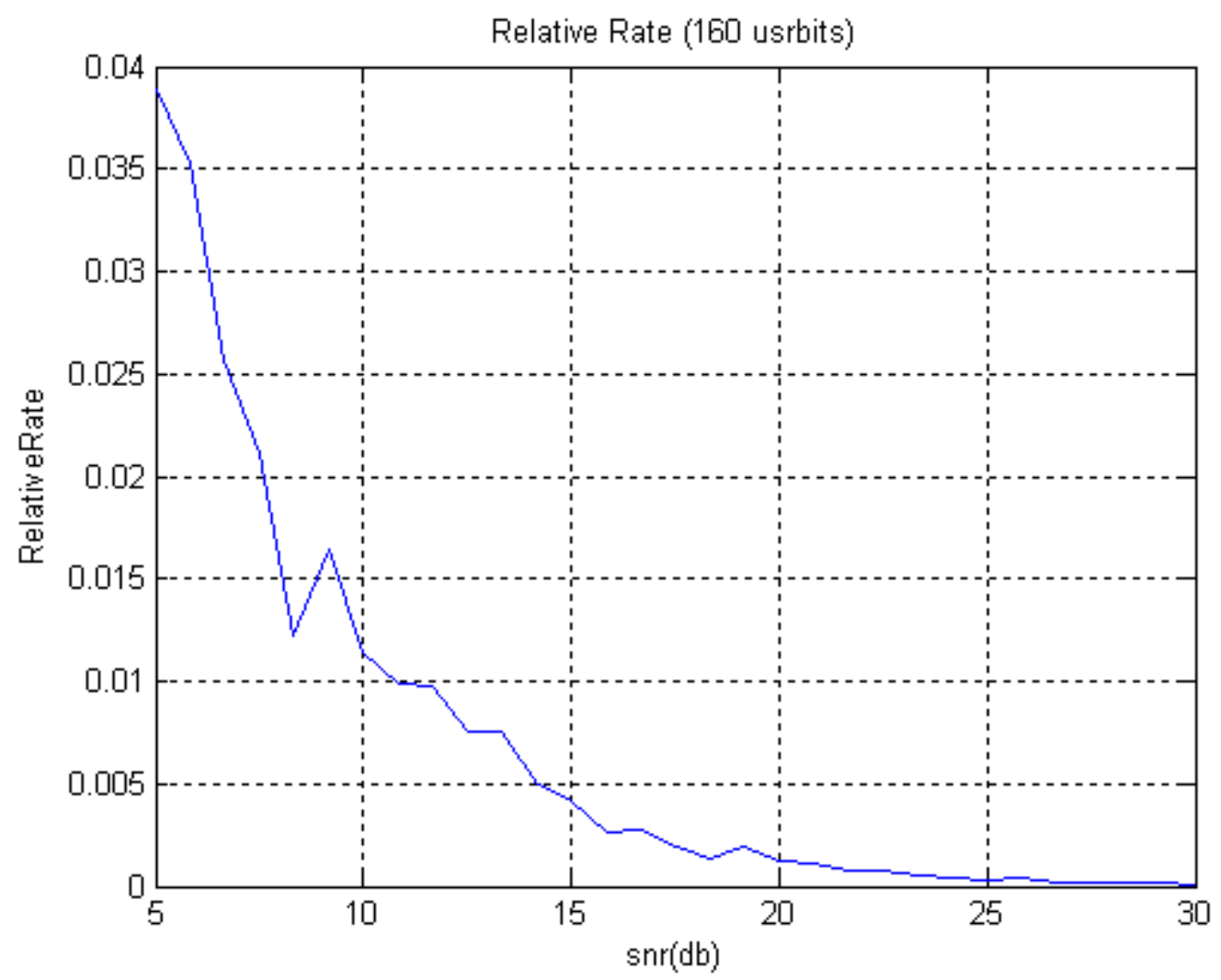

Fig.2. influence of SNR on relative error

In the simulation, the calibration matrix could effectively reduce the influence of the external factors. The relative error between the array output and the ideal output could be reduced. The calibration matrix improves the stability and accuracy of the system.

\section{Conclusion}

From the simulation and the analysis, it could be shown that the introduction of appropriate calibration algorithm could improve the accuracy of the array signal. And the calibration algorithm improves the system's signal to noise ratio, capacity performance and so on. In this paper, the calibration matrix was obtained based on the minimum mean square error criterion. The using of calibration matrix may reduce the error caused by the mismatch of the system effectively and improve the signal quality in wireless communication.

\section{References}

[1] Zhang, Yun Xia. "Dual-polarizaration Phased Antenna Array for Wind Profiler Radar." Modern Radar (2012).

[2] Li, Hailin, et al. "Radiated Power Classification and Polarization Control of Cylindrical Phased Array Antenna." Journal of Nanjing University of Aeronautics \& Astronautics (2013).

[3] Boskovic, N., B. Jokanovic, and A. Nesic. "Frequency scanning antenna array with enhanced side lobe suppression." Advanced Electromagnetic Materials in Microwaves and Optics (METAMATERIALS), 2014 8th International Congress on IEEE, 2014:67-69 
[4] Begaud, Xavier, et al. "Analytical model of a self-complementary connected antenna array on high impedance surface." Applied Physics A Materials Science \& Processing 115.2(2014):517-522.

[5] Zeng, Guoqi, et al. "Research on Pattern Synthesis for Low Side Lobe of Truncated Cone Conformal Phased Array Antenna." Journal of Convergence Information Technology (2013). 